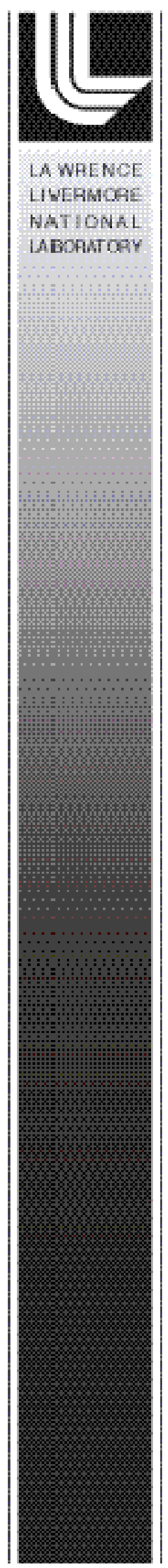

\title{
A 3-D Model of Signaling and Transport Pathways in Epithelial Cells
}

Andrew Quong,

Charles Westbrook

March 29, 2005 


\section{Disclaimer}

This document was prepared as an account of work sponsored by an agency of the United States Government. Neither the United States Government nor the University of California nor any of their employees, makes any warranty, express or implied, or assumes any legal liability or responsibility for the accuracy, completeness, or usefulness of any information, apparatus, product, or process disclosed, or represents that its use would not infringe privately owned rights. Reference herein to any specific commercial product, process, or service by trade name, trademark, manufacturer, or otherwise, does not necessarily constitute or imply its endorsement, recommendation, or favoring by the United States Government or the University of California. The views and opinions of authors expressed herein do not necessarily state or reflect those of the United States Government or the University of California, and shall not be used for advertising or product endorsement purposes.

\section{Auspices Statement}

This work was performed under the auspices of the U. S. Department of Energy (DOE) by the University of California, Lawrence Livermore National Laboratory (LLNL) under Contract No. W-7405-Eng-48. The project (02-ERD-027) was funded by the Laboratory Directed Research and Development Program at LLNL. 


\title{
FY04 LDRD Final Report \\ A 3-D Model of Signaling and Transport Pathways in Epithelial Cells \\ LDRD Project Tracking Code: 02-ERD-016 Andrew Quong, Principal Investigator Charles K. Westbrook
}

\begin{abstract}
A 3-dimensional computer model was developed to simulate the spatial and chemical evolution of calcium ions inside an array of human epithelial kidney cells. This is a prototype model, intended to develop a methodology to incorporate much more complex interactions of metabolic and other processes within many types of cells and lead to increased ability to predict cellular responses to disease as well as to chemical and biological warfare situations. Preliminary tests of the model are described.
\end{abstract}

\section{Project Background and Motivation}

The recent completion of the Department of Energy's Human Genome Project indicated the remarkable progress scientists have made in understanding the genetic basis of life. The successor to the Human Genome Project is the Genomes to Life Program, which has an even more ambitious goal, to understand life in a comprehensive and integrated manner. It includes a systems biology approach, the study of tissues as integrated systems rather than isolated parts, such as specific genes, proteins or chemical reactions.

This LDRD project has represented a beginning in a very new research direction, with the Genomes to Life approach in mind. The specific goal was to design and build a computational model of many of the integrated processes occurring in one class of cells, providing a multidisciplinary approach with biology, biochemistry, molecular biology, chemistry, physics, and computer science. LLNL has a long and impressive history of developing and applying multidisciplinary models to extremely complex processes in plasma physics, atmospheric chemistry and physics, underground water flows, and many others, taking advantage of the unique and powerful supercomputing resources at LLNL.

Ultimately, advanced computational models, based on sound chemical, physical and biological principles and new laboratory experiments, and running on the fastest supercomputers will permit scientists to visualize and understand the complex interactions and changes within a simulated family of cells. Past cell models have been relatively simple, treating cellular processes as if they were dimensionless electrical circuits, with a limited number of chemical reactions and 
other features included in the model. However, it is our eventual goal to be able to include a vast array of interconnected processes in a fully three dimensional set of simulations that could be used to predict a cell's response to different mixes of nutrients or exposure to a drug or a toxin from a pathogen.

In the present project, we have focused on a small part of such a massive computational undertaking, while at the same time keeping in mind the larger scope of a much more extended cell model. This initial step is intended to be a learning experience that will move us towards an eventual full-size realistic model. In this way, we can avoid short-sighted simplifications that would make model extensions more difficult in the future. At the same time, we can gain experience with the basic ideas of modeling cellular processes, including particularly localized chemical reactions imbedded in a domain in which chemical species are being transported throughout a number of contiguous cells.

For this effort, the problem of calcium ion transport within epithelial cells was selected. Epithelial cells line all of the body cavities in humans, including the lungs, digestive tract, and kidneys, and they are the barriers that protect the body from the external world and inhibit and control the movement of water, molecules and ions across these barriers. Thus a pathogen's first interaction with a human is with epithelial cells, so understanding these interactions could have important application to human health and protection against bioterrorist attacks. The epithelial cells in the kidney were selected as the specific human environment for study, since these cells are grown easily in culture and can be used in associated experimental studies. Another advantage is that the cells are roughly cubic, which lends themselves to easy numerical simulation in three spatial dimensions.

Successful completion of this project was expected to provide experience with modeling cellular processes and to provide new information about key functions of these cells, building on LLNL expertise in computational biology, supercomputing, biosecurity, and multiscale modeling of materials and chemical reactions. It is also possible to result in new insights into interactions between pathogens (viruses or bacteria) and human host cells. Eventually this type of modeling will likely result in advances in biological control and prevent diseases, as well as provide a basis for news initiatives in homeland security to detect and thwart any attempt at biological terrorism. This effort also included some experimental cell biology experiments with Michael Thelen of the LLNL Bioscience directorate as the task leader.

This model has been built on the previously existing ALE3D code, a computer code originally written for studying the detonation of high explosives. While detonations in high explosives and molecular transport in epithelial cells at first appear to be completely different, the nature of the computational modeling needs for these two classes of problems was found to be logically quite similar, but the enormous level of flexibility that was incorporated into the ALE3D code was found to be very helpful in addressing cellular processes, and relatively little modification was needed to apply it to models of cellular processes. 


\section{Nature of the Computational Problem and Results}

Nature has developed a highly regulated system for transport of ions and small molecules across the epithelial barrier that separates the surfaces of the body from the external environment. These systems contain regulatory elements controlled by external and internal signals. In order to understand the complexity of these systems, we developed a fully three-dimensional model of calcium signaling in epithelial cells. The model is based of a set of reaction diffusion equations that are solved on a large-scale finite-element code in three dimensions. We explicitly included the major compartments in the cell, such as the nucleus and endoplasmic reticulum. The model must allow for buffering of free $\mathrm{Ca}^{2+}$, calcium induced calcium release (CICR), and for explicit inclusion of mobile buffers, mimicking the dyes used in the experiments. Additionally, we included a realistic representation of the gap junctions that have a significant effect on $\mathrm{Ca}^{2+}$ wave propagation.

An experimental view of a number of epithelial kidney cells is shown in Figure 1a, with the cell membranes appearing white in the figure. The overall regularity in the cell sizes and shapes is evident, and a preliminary computational view of the same group of cells is shown in Figure 1b. At this point, the code was modified to use some of the advanced grid generation capabilities of the ALE3D code system, and the artificially rigid "cells" in Figure $1 \mathrm{~b}$ were replaced by a much more realistic spatial formulation as shown in Figure 2.

Experimental $\mathrm{Ca}^{2+}$ concentrations in epithelial cells are shown in Figure $3 a$, in which the calcium is indicated by a red dye that was bound to the calcium. The calcium was initially injected into a single cell, and the results in Figure 3a show that the calcium has spread to some of the adjacent cells. A computational view of the same problem is shown in Figure 3b, which shows the initial conditions in the matching computational problem. The flexibility of the ALE3D grid-generation formulation is evident from this picture.

In simulating the transport of calcium ions in kidney epithelial cells, the model includes the movement of the small messenger inositol-1,4,5-triphosphate (called $\mathrm{IP}_{3}$ ) molecules which coordinate the release of calcium waves. The waves, lasting several seconds each, are believed to be an important signaling mechanism as they move within and across epithelial cells. By binding to receptors, which are folded proteins located within the cell, $\mathrm{IP}_{3}$ molecules trigger the release of calcium.

Although there have been other models of calcium waves, they have been limited to one or two spatial dimensions, so this represents the first fully $3 \mathrm{D}$ model that is consistent with experimental data and is based upon valid physiology and chemistry. One goal of this model is accurately to predict where calcium is stored within the cell prior to being released as part of a wave. Calcium ions are often found sequestered in certain organelles, specialized cell 
parts analogous to organs. This problem was selected for study in companion experiments that were carried out by Michael Thelen in the Biosciences Directorate.

Another view of a calcium wave is shown in Figure 4. In addition to showing the propagation of calcium ions in general, the figure indicates locations in the epithelial cell boundary with the outside environment where information passes into the cell layer (indicated in red) and the randomly chosen $\mathrm{IP}_{3}$ receptors located on the boundaries of the endoplasmic reticulum, shown in black. The effect of randomness in these locations on wave propagation is one feature that has given rise to a stochastic simulation model for this process.

Generation of transient $\mathrm{Ca}^{2+}$ waves is a ubiquitous motif in intracellular signaling, utilized by processes such as muscle contraction and oocyte fertilization. These ions are pumped from one region of a cell to another and diffuse across each cell and between cells. The development of transport algorithms, with a suitable amount of stochastic simulation features, coupled with kinetics of reactions, provides a challenge for the mathematics and computer science of these simulations, and a great deal of time and effort in this project have been concentrated in these areas. The sheer size of these three dimensional diffusion-reaction problems requires massively parallel computing resources, and as additional complexity is introduced into these problems, the computational challenges are expected to increase.

\section{Project Completion}

This project completed two years with excellent research, innovation in a number of important new subject areas, and a computational environment that suggests that the concept of cell modeling has been demonstrated and that new functionality would eventually be feasible. However, two key participants then were offered and accepted faculty positions outside LLNL, including the principal investigator of this project, and both soon departed. The resulting lack of leadership and overall manpower made it possible only to bring the project to a sensible, organized conclusion and has made the next steps for this work questionable. At present, the lack of knowledgeable researchers has required us to en this project coincident with the completion of the LDRD project, until a new PI is identified to determine the next technical steps.

The success of the concept of providing system-level simulation has certainly been demonstrated, a success which could not have been anticipated with much confidence at the origin of the project. Computational physics projects with similar scope have been successful at LLNL in the past, and a similar demonstration for biomolecular signaling and other multicellular processes has been achieved. There are many conceptual gaps in our understanding of intracellular and intercellular propagation that would stand in the way of the next steps in this process. In many cases the couplings between 
subsystems are still unknown, but a model of this type could perhaps identify the data that are needed to make those next steps.

There are many diffusion-reaction systems operating at the same time as the calcium intracellular signaling system, some of which operate independently of the calcium signaling and some which interact in a non-linear way with it. These systems and their interactions will influence the present set of solutions until all of the important couplings are included. While the present formulation is instructive and provides some experience in dealing with the computational problems involved, the additional complexity will make the solutions much more demanding in computational terms, and it will also mean that the computed results will not provide reliable insights until the full problems can be considered. That will require significant additional time, researchers, and resources.

\section{Conclusions}

The goal of this project was to develop a computer model capable of simulating reaction/diffusion problems within a class of cells found in many parts of the human body, including the three-dimensional nature of these systems. This task required the massively parallel computer resources available at LLNL and which will be required for similar simulations of the even more complex problems we hope to pursue in the future. While this work has provided additional insight into the propagation mechanisms for calcium within the epithelium, a more significant accomplishment is the demonstration of the concept of performing realistic biochemical simulations with even greater complexity and biological realism in the future. Some understanding of the further computational science developments that will be required has been developed. Several journal papers will be published in part or entirely as results of this LDRD project. 


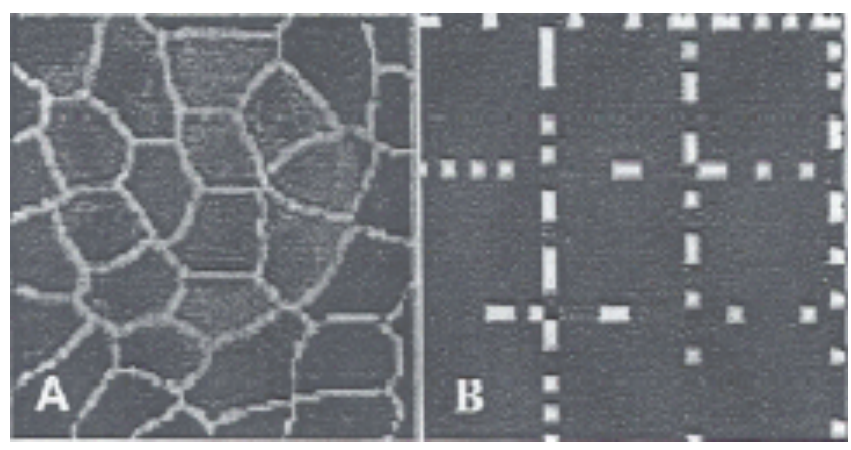

Figure 1

Experimental image ofCa in kidney epithelium (left) and preliminary simulation view (right) in simplified geometry.

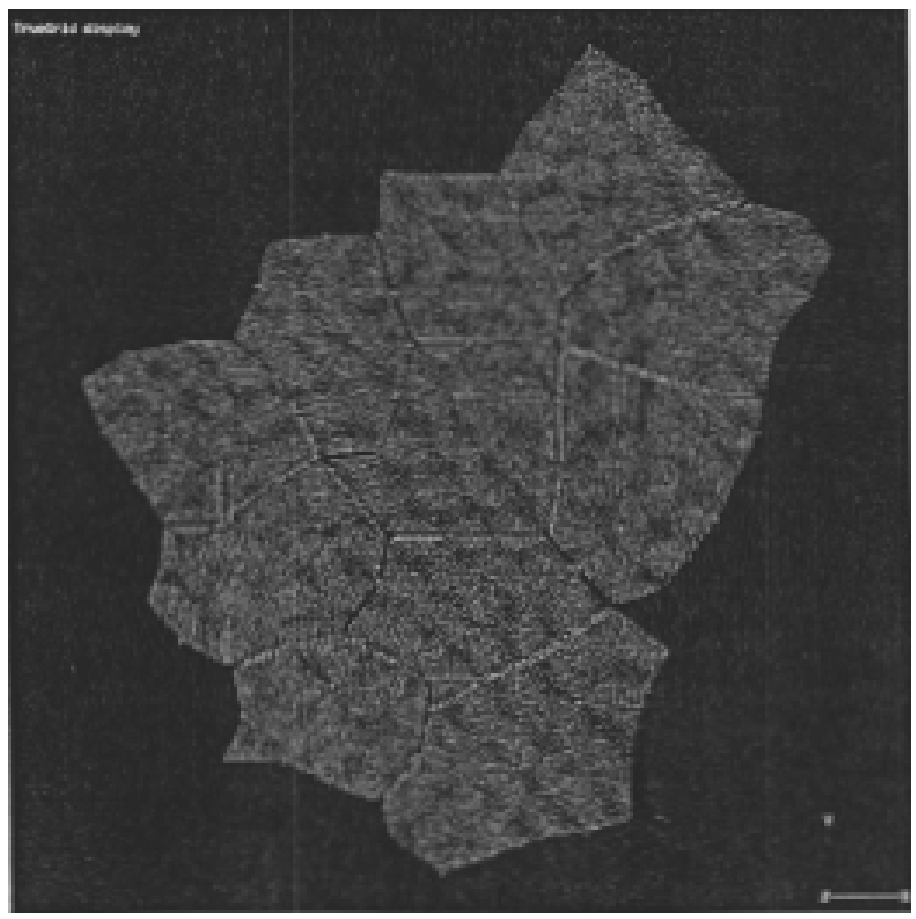

Figure 2

Computational mesh generated from the experimental image in Figure 1. 



\section{Figure 3}

Code simulation of the flow of calcium ions compares favorably to experimental images of calcium ion waves. (a) An image from confocal microscopy captures a wave of calcium ions (bound to a red dye) that began in one epithelial cell and spread to its neighbors. (Image reprinted with permission from Science 284, May 28, 1999, 1529. Copyrighted 1999, American Association for the Advancement of Science.) (b) The Livermore code simulates a wave of calcium ions (in red) triggered by the cell located in the center. The simulated cells measure about 25 micrometers across. The white lines represent cell membranes and the missing portions indicate gaps in the membrane where calcium ions pass from one cell to another. 

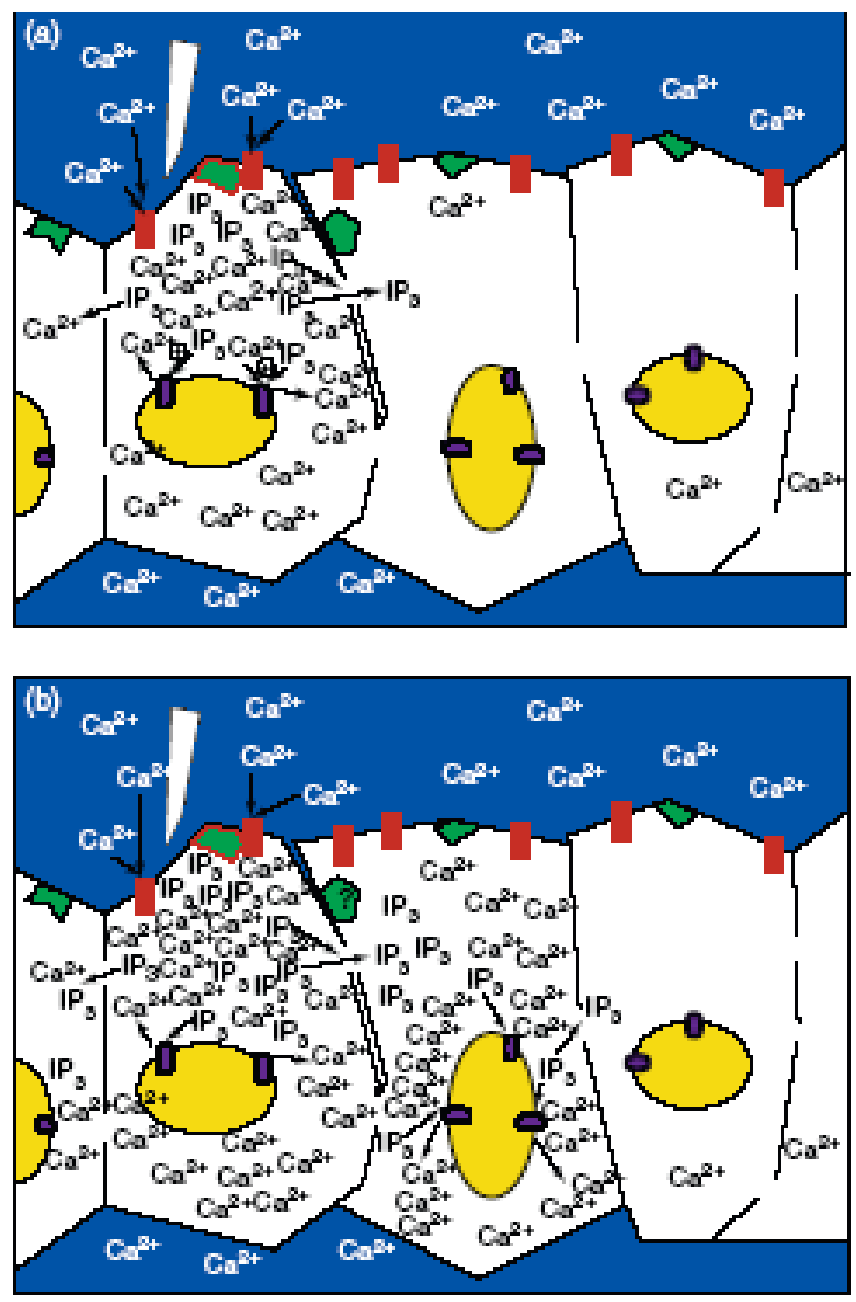

Figure 4

Waves of calcium ions are believed to be an important signaling mechanism as they move within and across epithelial cells. The waves are coordinated by inositol-1,4,5-triphosphate $\left(\mathrm{IP}_{3}\right)$ which binds to certain cell receptors. The diagram illustrates the flow of calcium and $\mathrm{IP}_{3}$ molecules from (a) one cell to (b) its neighbors. 